\title{
MSH5, a novel MutS homolog, facilitates meiotic reciprocal recombination between homologs in Saccharomyces cerevisiae but not mismatch repair
}

\author{
Nancy Marie Hollingsworth, ${ }^{1}$ Lisa Ponte, and Carol Halsey \\ Department of Biochemistry and Cell Biology, State University of New York, Stony Brook, Stony Brook, New York \\ 11794-5215 USA
}

\begin{abstract}
Using a screen designed to identify yeast mutants specifically defective in recombination between homologous chromosomes during meiosis, we have obtained new alleles of the meiosis-specific genes, HOP1, RED1, and MEK1. In addition, the screen identified a novel gene designated MSH5 (MutS homolog 5). Although Msh5p exhibits strong homology to the MutS family of proteins, it is not involved in DNA mismatch repair. Diploids lacking the MSH5 gene display decreased levels of spore viability, increased levels of meiosis I chromosome nondisjunction, and decreased levels of reciprocal exchange between, but not within, homologs. Gene conversion is not reduced. Msh5 mutants are phenotypically similar to mutants in the meiosis-specific gene MSH4 (Ross-Macdonald and Roeder 1994). Double mutant analysis using msh4 msh5 diploids demonstrates that the two genes are in the same epistasis group and therefore are likely to function in a similar process-namely, the facilitation of interhomolog crossovers during meiosis.
\end{abstract}

[Key Words: MSH5; yeast; MutS homolog; recombination]

Received March 9, 1995; revised version accepted June 6, 1995.

A unique problem confronting sexually reproducing organisms is how to maintain the parental chromosome number in offspring produced by fertilization. The solution to this problem is to reduce the number of chromosomes in germ cells by half in a very precise manner such that each gamete receives one, and only one, member of each chromosome pair. Fertilization then restores the diploid chromosome number. The reduction in chromosome number is accomplished by meiosis, during which a single round of chromosome replication is followed by two rounds of chromosome segregation. Failures in meiosis result in gametes that are chromosomally imbalanced or aneuploid.

Proper segregation of homologs at the first meiotic division requires that the chromosomes align correctly on the metaphase plate. For this alignment to occur, the homologs must be physically connected by crossovers or chiasmata (for review, see Hawley 1987). These crossovers must occur in the context of the synaptonemal complex (SC), the proteinaceous structure formed when homologous chromosomes become physically associated, to function in segregation (Engebrecht et al. 1990).

To understand how functional chiasmata are gener-

${ }^{1}$ Corresponding author. ated in the presence of SCs, it is necessary to first define the proteins involved both in synapsis (i.e., SC formation) and recombination. Antibodies directed against purified SCs have enabled the isolation of genes encoding SC components in mammals (Meuwissen et al. 1992). In yeast a number of different genetic screens have been used to identify genes important for recombination, synapsis, or both (Petes et al. 1991). One of these screens detects mutants specifically defective in recombination between homologs during meiosis (Hollingsworth and Byers 1989|. The basis of this approach is to look for mutants that are unaffected for reciprocal recombination within a chromosome, but reduced for recombination between homologs. Because several genes have been shown to be necessary for both intra- and interchromosomal recombination (MER1, SPO11, and RAD50), the assumption is that the same recombination machinery mediates both types of events (Wagstaff et al. 1985; Engebrecht and Roeder 1989; Gottlieb et al. 1989). In theory, therefore, this screen should detect mutants defective in synapsis, as well as in processes that facilitate crossovers specifically between homologous chromosomes.

This screen was used successfully to isolate $H O P 1$, a structural component of meiotic chromosomes in yeast, which is necessary for homolog synapsis (Hollingsworth 
and Byers 1989; Hollingsworth et al. 1990). Subsequent reconstruction experiments indicated that mutations in two other genes, RED1 and MEK1, also specifically reduce interhomolog recombination and, therefore, should have been detected (Rockmill and Roeder 1990; B. Rockmill, pers. comm.). Like HOP1, RED1 is required for synapsis (Rockmill and Roeder 1990). MEK1 encodes a meiosis-specific kinase required for complete $\mathrm{SC}$ formation (Rockmill and Roeder 1990; Leem and Ogawa 1992). Because only a single allele of $H O P 1$ was identified in the original screen, the screen was repeated in an attempt to find additional genes that may be important for synapsis and/or interhomolog recombination.

This paper reports the discovery of a novel MutS homolog designated MSH5 (Muts homolog 5). MutS homologs function in mismatch repair in a variety of different organisms, including bacteria, yeast, and humans (for review, see Modrich 1991). In addition, mutS is required to prevent partially diverged sequences from undergoing recombination in bacteria (Rayssiguier et al. 1989|. We demonstrate here that, unlike mutS and the yeast MSH1 and MSH2 family of MutS homologs, the MSH5 gene plays no role in DNA mismatch repair. Instead, $\mathrm{MSH} 5$ appears to facilitate reciprocal crossovers between homologs, presumably to prevent nondisjunction at the first meiotic division.

\section{Results}

A screen for mutants specifically defective in meiotic interchromosomal recombination identifies new alleles of HOP1, RED1, and MEK1, as well as MSH5, a novel MutS homolog

To find mutants defective specifically in meiotic interhomolog recombination, the chromosome III disomic haploid karC2-4 (Table 1) was mutagenized. A total of 9855 colonies were assayed for a change in the segregation pattern of chromosome III, which is indicative of a defect in reciprocal recombination between homologs. At the same time the screen required that efficient reciprocal intrachromosomal recombination still occur (Hollingsworth and Byers 1989). In this assay a correlation has been observed previously between a decrease in meiotic interhomolog recombination and an increase in the fraction of equationally segregating chromosomes in the single-division spo13 meiosis. Mutants exhibiting increased levels of equational segregation in the spo13 disomic haploids were tested further for their effects on spore viability in SPO13 diploids. Defects in reciprocal recombination between chromosomes reduce spore viability in SPO13 meiosis because of the requirement for crossovers for proper chromosome disjunction at meiosis I. Mutants exhibiting mitotic phenotypes such as sensi-

Table 1. Saccharomyces cerevisiae strains

\begin{tabular}{|c|c|}
\hline Strain & Genotype \\
\hline \multicolumn{2}{|c|}{ A. Disomic haploids } \\
\hline karC2-4 & MAT $\alpha$ cdc10-2 LEU2: pNH18 (URA3 CYH2) HIS4 trp1 ura3-52 can1 cyh2 spo13-1 ade2-1 \\
\hline B31-89 & $\begin{array}{l}\text { MATa CDC10 leu2 } \\
\text { karC2-4 but msh5-1 }\end{array}$ \\
\hline \multicolumn{2}{|c|}{ B. $S K 1$ background ${ }^{\mathrm{a}}$} \\
\hline \multicolumn{2}{|c|}{ NH144 MATa leu2-k HIS4 arg4-nsp ura3 lys2 ho::LYS2 } \\
\hline \multicolumn{2}{|c|}{$\begin{array}{ll} & \text { MATa leu2Ahisg his4-x ARG4 ura3 lys2 ho::LYS2 } \\
\text { NH145 } & \text { NH144 but msh5::URA3 }\end{array}$} \\
\hline \multicolumn{2}{|r|}{$\begin{array}{c}\overline{m s h 5:: U R A 3} \\
\text { NH144 but } \frac{m s h 5:: U R A 3 \text { msh4::LEU2 }}{\text { msh5::URA3 msh4::LEU2 }}\end{array}$} \\
\hline \multicolumn{2}{|c|}{ C. BR background ${ }^{a}$} \\
\hline NH156 & MATa LEU2 his4 ade2 MET13 trp5 cyh3 ade6 ura3 spo13Aura3 \\
\hline NH157 & $\begin{array}{l}\overline{M A T \alpha} \text { leu2 } H I S 4 \\
\text { NH156 but } \frac{\text { ade2 met13 }:: U R A 3}{\text { msh5::URA3 }}\end{array}$ \\
\hline \multicolumn{2}{|c|}{$\begin{array}{l}\text { PS593 } \\
\text { PS593/190 }\end{array}$} \\
\hline \multicolumn{2}{|c|}{ E. Miscellaneous strain background } \\
\hline \multirow[t]{3}{*}{ NH111 } & MAT $\alpha$ LEU2 HIS4 his7 trp1 msh5-1 ura3-52 CAN1 CYH2 spo13-1 \\
\hline & 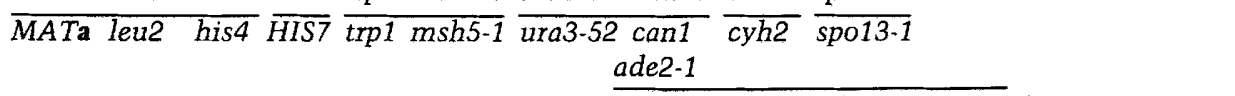 \\
\hline & ade2-1::pNH164 (ADE2 SPO13) \\
\hline
\end{tabular}

Strains are grouped in isogenic sets (A-E).

${ }^{\text {a }}$ From P. Ross-MacDonald. 
tivity to the DNA-damaging agent, methyl methane sulfonate (MMS), or slow growth were excluded.

Eleven new mutants were isolated, which displayed increased levels of equational segregation of chromosome III in spo13 disomic haploids and reduced spore viability in SPO13 diploids (Table 2). All of the mutations are recessive. Complementation tests using spore viability as an assay revealed that of these 11 mutants, 5 carry alleles of $M E K 1,2$ carry alleles of $H O P 1$, and 3 carry alleles of $R E D 1$. Subsequent experiments demonstrated that the remaining mutant, $m s h 5-1$, identifies a novel MutS homolog that we designate MSH5 (see below).

msh5 decreases spore viability in SPO13 diploids and causes increased meiosis I nondisiunction

A number of meiotic mutants have been described recently that moderately reduce spore viability in SPO13 strains (zip1, med1, msh4) (Sym et al. 1993; Rockmill and Roeder 1994; Ross-Macdonald and Roeder 1994). Diploids homozygous for msh5-1 fall into this class, producing $37 \%$ viable spores (Table 2 ). To determine whether this level of spore viability represents the null phenotype for $\mathrm{MSH} 5$, an insertion allele was created using the cloned gene (see Materials and methods). The URA3 gene was inserted into a unique BglII site within the MSH5-coding sequence (Fig. 3). This insertion creates a truncated Msh5 protein, removing two highly conserved functional domains (see below). The

Table 2. Assay of equational segregation in spol3 disomic haploids and spore viability in SPO13 diploids for mutants detected by the screen

\begin{tabular}{lccc}
\hline Genotype & $\begin{array}{l}\text { Disomic } \\
\text { haploid } \\
\text { strain }\end{array}$ & $\begin{array}{l}\text { Equational } \\
\text { segregation of } \\
\text { chromosome } \\
\text { III }(\%)\end{array}$ & $\begin{array}{l}\text { SPO13 } \\
\text { diploid } \\
\text { (\% spore } \\
\text { viability })^{\mathrm{a}}\end{array}$ \\
\hline+ & karC2-4 & 17 & $92(72)$ \\
mek1-974 & A9-74 & 43 & $48(103)$ \\
mek1-3382 & A33-82 & 87 & $1(18)$ \\
mek1-4113 & B41-13 & 90 & $10(20)$ \\
mek1-5069 & B50-69 & 70 & $12(22)$ \\
mek1-5994 & B59-94 & 83 & $57(38)$ \\
hop1-1 & $2-26$ & 95 & $1(52)$ \\
hop1-742 & B7-42 & 100 & $\sim 1^{\mathrm{c}}$ \\
hop1-348 & B3-48 & 100 & $\sim 1^{\mathrm{c}}$ \\
red1-2353 & B23-53 & 88 & $1(18)$ \\
red1-867 & A8-67 & 78 & $5(10)$ \\
red1-6183 & B61-83 & 82 & $\sim 1^{\mathrm{c}}$ \\
msh5-1 & B31-89 & 84 & $37(485)$ \\
\hline
\end{tabular}

a The value in parentheses represents the number of asci dissected. SPO13 mutx spore viability was determined by isolating $M A T \mathbf{a}$ and $M A T \alpha$ haploid derivatives from each mutant disomic haploid, integrating the SPO13 gene into the MATa derivative, crossing the two haploids, and dissecting the asci obtained from the sporulated diploids.

${ }^{b}$ hop 1-1 data from Hollingsworth and Byers (1989).

${ }^{\mathrm{c}}$ Qualitative results obtained by random spore analysis.

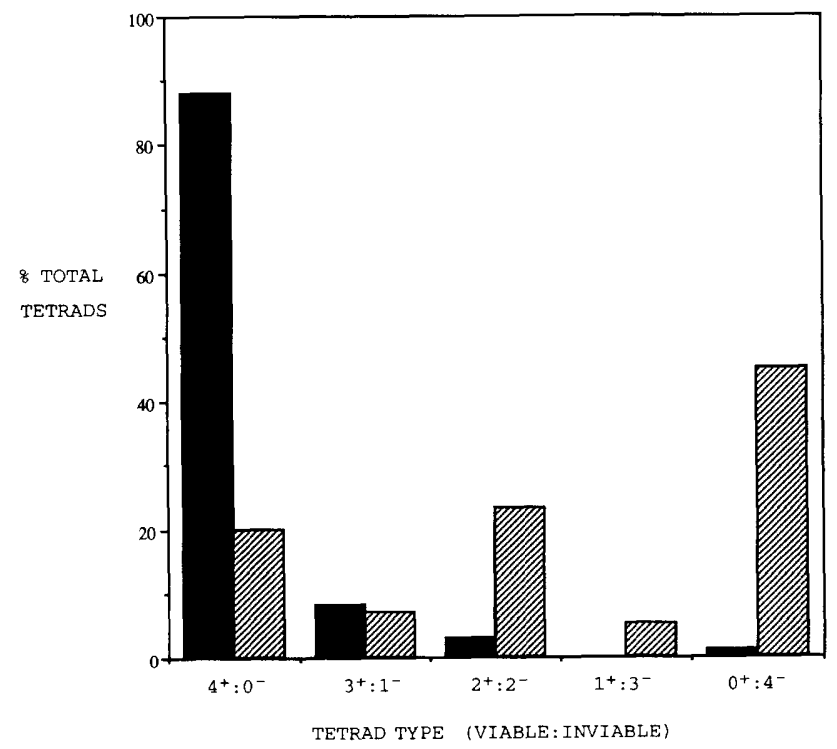

Figure 1. Comparison of the pattern of viable spores within tetrads in the SK1 isogenic diploids NH144 (MSH5/MSH5) and NH145 (msh5::URA3/msh5::URA3). For NH144, 337 tetrads were dissected. For NH145, 1370 tetrads were dissected. (Solid bar) MSH5; (hatched bar) msh5::URA3.

msh5::URA3 allele was then substituted for the wildtype copy in two different strain backgrounds-A364A and SK1. In both backgrounds the level of spore viability observed was similar to the $37 \%$ found for $m s h 5-1(50 \%$, 146 tetrads for the A364A background; and 38\%, 1370 tetrads, for the SK1 background).

The observation that spo13 can rescue the msh5-1 spore lethality suggests that the spore inviability conferred by msh5-1 is attributable to the mis-segregation of homologs at the first meiotic division. The single-division spo13 meiosis has been shown to rescue a large number of recombination-deficient mutants (Petes et al. 1991). The msh5-1 SPO13 diploid produces $37 \%$ viable spores (485 tetrads). In the isogenic msh5-1 spo13 diploid, spore viability is improved to $66 \%$ (327 dyads). This value is also an improvement over the $22 \%$ (660 dyads) viability observed in the isogenic MSH5 spo13 diploid, consistent with previous observations that defects in recombination improve spore viablity in spo13 meiosis.

Further evidence for a Meiosis I defect for msh5 was obtained by analyzing the pattern of spore viability in tetrads produced by the msh5::URA3 SPO13 diploid $\mathrm{NH} 145$. The pattern of spore death in NH145 is nonrandom, with an excess of two- and zero- viable spore tetrads at the expense of tetrads with four viable spores (Fig. 1). This pattern is indicative of meiosis I nondisjunction (Fig. 2). The tetrads with two viable spores result when one or more pairs of homologs segregate to the same pole. The spores that survive are disomic for the missegregated homologs. Tetrads with zero viable spores result when two or more pairs of homologs disjoin to opposite poles.

It is possible in S. cerevisiae to show definitively that 
A.

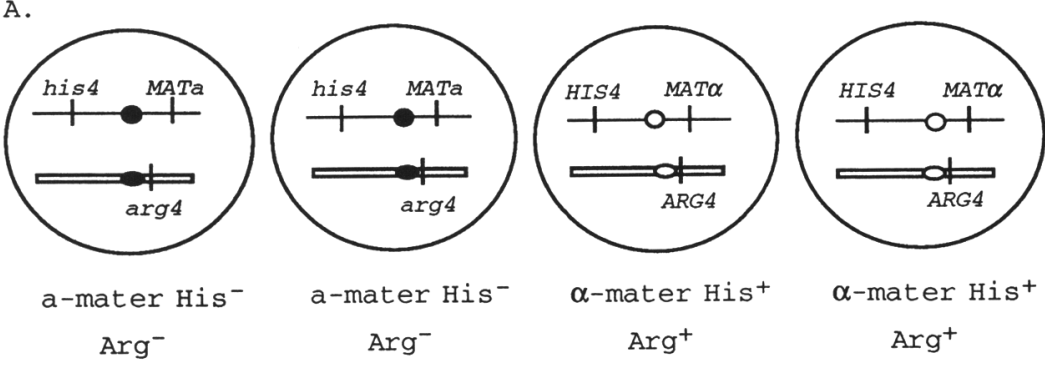

B.

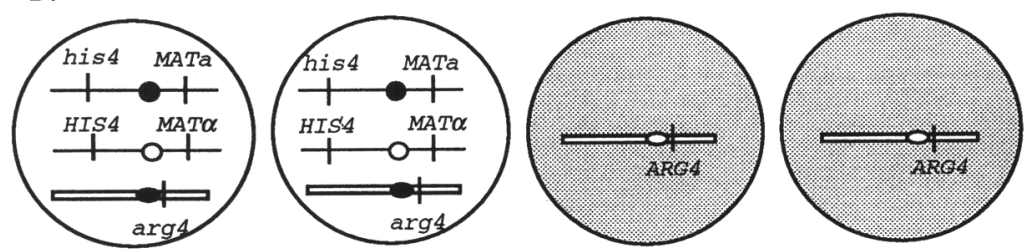

Non-mater $\mathrm{His}^{+}$Non-mater $\mathrm{His}^{+}$

$\mathrm{Arg}^{-} \mathrm{Arg}^{-}$

C.
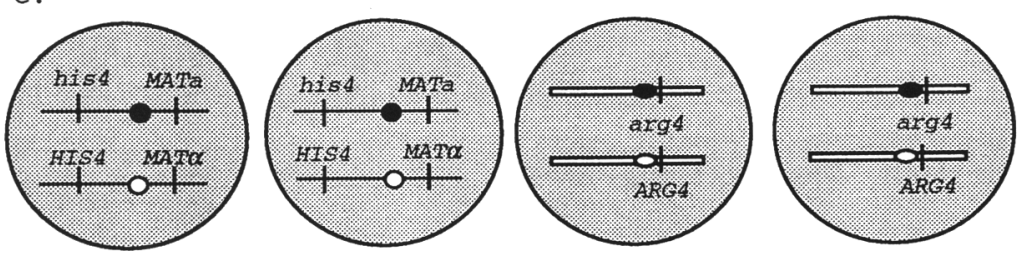

Figure 2. Diagram of tetrads undergoing, no nondisjunction $(A)$; nondisjunction of chromosome III $(B)$; nondisjunction of chromosome III and chromosome VIII $(C)$. No recombination is assumed. Sister centromeres are indicated by white or shaded circles. Shaded spores are inviable. meiosis I nondisjunction is occurring by taking advantage of the codominant MATa and MAT $\alpha$ alleles present on chromosome III. As depicted in Figure 2, meiosis I nondisjunction of chromosome III results in tetrads with two viable spores, where both spores are nonmaters because of the presence of both MATa and MAT $\alpha$ information. Of 308 two-viable spore tetrads analyzed for NH145, 15.3\% contained two nonmating spores, demonstrating that in the msh5::URA3 diploid, chromosome III is mis-segregating frequently.

Analysis of the segregation of the chromosome VIII centromere-linked marker arg4 indicates that other chromosomes besides chromosome III are mis-segregating as well. If spore death is attributable to nondisjunction of a pair of homologous chromosomes to the same pole at meiosis I, equational segregation of the properly disjoined homologs at meiosis II will result in the two suriviving spores containing sister chromatids (such spores are designated sister spores). Assuming that no recombination exists between arg 4 and the centromere, sister spores should have the phenotype $\mathrm{Arg}^{+}: \mathrm{Arg}^{+}$or $\mathrm{Arg}^{-}: \mathrm{Arg}^{-}$(Fig. 2). We observed that the majority $(83 \%$ ) of 135 two-viable spore tetrads scored for NH145 are comprised of chromosome VIII sister spores. This difference is significantly different from the $50 \%$ predicted if spore death were random $\left(\chi^{2}, P<0.001\right)$. A similar statistically significant bias for chromosome III sister spores in two-viable spore tetrads where chromosome III had properly segregated was also observed (data not shown). The effect of msh5::URA3 on chromosome nondisjunction therefore appears to be general.

Another type of chromosome mis-segregation event that may occur is precocious separation of sister chromatids (PSSC), where sister chromatids of one homolog fall apart prior to meiosis I and then segregate randomly to either pole. In some cases, mutants that cause an increase in meiosis I nondisjunction also result in an increase in PSSC (Miyazaki and Orr-Weaver 1992; Rockmill and Roeder 1994). Precocious sister chromatid separation of chromosome III is manifested by three-viable spore tetrads in which one spore is a nonmater, one is an a-mater, and one is an $\alpha$-mater. Of 87 three-viable spore tetrads analyzed for NH145, 3 exhibited this pattern. In the same strain background, Sym and Roeder (1994) observed no chromosome III disomic spores of 57 threeviable spore tetrads. We conclude, therefore, that whereas the major defect caused by the absence of MSH5 is an increase in nondisjunction of homologous chromosomes at the first meiotic division, other types of missegregation events may be elevated as well. 
msh5 decreases meiotic reciprocal exchange between homologs but does not reduce gene conversion

To test whether crossing-over is affected by the absence of $M S H 5$, intervals on chromosome III and chromosome VII were assayed by ascus dissection for recombination. A pair of isogenic spo13 diploids, NH156 and NH157, were constructed that are homozygous for $\mathrm{MSH} 5$ and $m s h 5:: U R A 3$, respectively. As discussed earlier, the single-division meiosis conferred by spo13 eliminates the need for crossovers to ensure proper reductional segregation of homologs at the first meiotic division. One can therefore analyze recombination in spo13 strains without having to account for potential bias introduced by poor spore viability. As is the case with the other genes identified by this screen, HOP1, RED1, and MEK1 (Hollingsworth and Byers 1989, Rockmill and Roeder 1990, 1991), the msh5::URA3 and msh5-1 mutations reduce, but do not eliminate, reciprocal exchange (Table 3; N.M. Hollingsworth, unpubl.). The msh5::URA3 mutation resulted in a statistically significant reduction in recombination of either two- or threefold in five intervals on two different chromosomes (Table 3). A similar reduction in recombination was observed for the HIS4-MAT interval when four-viable spore tetrads from the SK1 SPO13 diploids NH144 and NH145 were compared (Table 3).

Our hypothesis is that the high levels of nondisjunction observed in msh5 diploids are attributable to the reduction in crossing-over. If this is the case, then the chromosome III disomic spores found in the two viable spore tetrads of $\mathrm{NH} 145$ should both be heterozygous at HIS4 and therefore prototrophic for histidine (Fig. 2). Of the 47 tetrads observed to have undergone meiosis I nondisjunction of chromosome III, 2 contained one histidine prototrophic and one histidine auxotrophic spore. These spores could arise either by recombination between HIS 4 and the centromere or by gene conversion of the HIS4 allele to his4. Given the lack of a centromere-linked marker and the high frequency of conversion at HIS4 in this strain (see below), we could not distinguish between these two possibilities. Further work is therefore necessary to confirm whether there is a mechanistic link in msh5 mutants between crossing-over and nondisjunction.

HOP1, RED1, and MEK1 mutants not only reduce crossing-over between chromosomes, they also reduce

Table 3. Effect of msh5::URA3 on reciprocal recombination between homologs

\begin{tabular}{|c|c|c|c|c|c|c|c|}
\hline \multirow[b]{2}{*}{ Strain $^{a}$} & \multirow{2}{*}{\multicolumn{2}{|c|}{ Genotype }} & \multicolumn{5}{|c|}{ Intergenic distance $(\mathrm{cM})^{\mathrm{b}}$} \\
\hline & & & HIS4-LEU2 & $L E U 2-M A T$ & MET13-TRP5 & TRP5-CYH3 & $C Y H 3-A D E 6$ \\
\hline \multirow[t]{2}{*}{ NH156 } & MSH5 & spo13 & 13 & 38 & 47 & 24 & 19 \\
\hline & $\overline{M S H 5}$ & spo13 & & & & & \\
\hline \multirow[t]{3}{*}{ NH157 } & \multicolumn{2}{|c|}{ msh5::URA3 spo13 } & $7(0.5)^{\mathrm{c}}$ & $12|0.3|$ & $15(0.3)$ & $6(0.3)$ & $6(0.3)$ \\
\hline & \multicolumn{2}{|c|}{$\overline{\text { msh5::URA3 spo13 }}$} & & & & & \\
\hline & & & HIS4-MAT & & & & \\
\hline \multirow[t]{2}{*}{ NH144 } & \multirow{2}{*}{\multicolumn{2}{|c|}{$\frac{M S H 5 \quad M S H 4}{M S H 5 \quad M S H 4}$}} & 37 & & & & \\
\hline & & & & & & & \\
\hline \multirow[t]{2}{*}{ NH145 } & \multicolumn{2}{|c|}{ msh5::URA3 MSH4 } & $13(0.3)$ & & & & \\
\hline & \multicolumn{2}{|c|}{$\overline{m s h 5:: U R A 3 ~ M S H 4}$} & & & & & \\
\hline \multirow[t]{2}{*}{ NH155 } & \multicolumn{2}{|c|}{ msh5::URA3 msh4::LEU2 } & $10(0.3)$ & & & & \\
\hline & \multicolumn{2}{|c|}{$\overline{m s h 5:: U R A 3 \mathrm{msh} 4:: L E U 2}$} & & & & & \\
\hline
\end{tabular}

${ }^{a} \mathrm{NH} 156$ and $\mathrm{NH} 157$ are isogenic. $\mathrm{NH} 144, \mathrm{NH} 145$, and $\mathrm{NH} 155$ are isogenic with the SKl background.

${ }^{\mathrm{b}}$ Map distances for the spo13 strains were calculated by the formula

$$
\frac{\left(2 \times \mathrm{SCO}_{\mathrm{e}}\right)+\mathrm{SCO}_{\mathrm{r}}+6(\text { four strand DCO })}{\text { Total dyads }} \times \frac{100}{2}=\mathrm{cM}
$$

where $\mathrm{SCO}_{\mathrm{e}}$ and $\mathrm{SCO}_{\mathrm{r}}$ are single crossovers that have segregated equationally and reductionally, respectively. For the SPO13 diploids, the following formula was used (Perkins 1949):

$$
\frac{(T+6 \mathrm{NPD})}{\text { Total tetrads }} \times \frac{100}{2}=\mathrm{cM}
$$

For NH156, 99 dyads were analyzed for chromosome III markers and 104 dyads were analyzed for chromosome VII markers. For NH157, 104 dyads were analyzed for chromosome III and 115 for chromosome VII. For NH144, NH145, and NH155, 279, 229, and 65 tetrads were examined, respectively.

${ }^{c}$ Numbers in parenthesis represent the ratio of the msh5 map distance over the wild-type distance. All of the mutant values differ significantly from wild type by $\chi^{2}$ analysis $(P<0.001)$. 
gene conversion (Hollingsworth and Byers 1989; Rockmill and Roeder 1990, 1991; Leem and Ogawa 1992). Using isogenic diploids heteroallelic for ade2, ura3, or leu2, we assayed mitotic and meiotic gene conversion in MSH5, msh5-1, and msh5::URA3 diploid strains by selection for the formation of prototrophs. No significant differences between the msh 5 diploids and wild type were observed (N.M. Hollingsworth, L. Ponte, and C. Halsey, unpubl.). In addition, gene conversion was assayed in NH144 and NH145 at three loci (HIS4, ARG4, and $L E U 2$ ) by dissection (Table 4). A statistically significant increase in gene conversion was observed at $A R G 4$ in the msh5::URA3 diploid. In contrast, no effect was observed for msh5::URA3 on the conversion frequency at HIS4 or LEU2. MSH5 is therefore phenotypically distinct from $H O P 1, R E D 1$, and $M E K 1$ in that it does not generally reduce interhomolog recombination but does reduce reciprocal exchange.

\section{Cloning and mapping of MSH5 identifies it as a new gene}

To determine whether msh5-1 identifies a new gene, the MSH5 gene was cloned and mapped (see Materials and methods). Hybridization to an overlapping set of yeast genomic fragments mapped the cloned piece of DNA to chromosome IV. Meiotic mapping confirmed that the MSH5 gene is located $1.9 \mathrm{cM}$ from CDC36 on chromosome IV. This map position does not coincide with that of any previously identified genes.

Various subclones of the original cloned insert were tested for their ability to complement the spore inviability of the msh5-1 diploid NH111. The smallest complementing piece is a $4.7-\mathrm{kb}$ HindIII-SphI fragment contained in pNH181. pNH181 restores spore viability in $\mathrm{NH} 111$ from $50 \%$ with the vector YCp50 (48 tetrads) to 92.5\% (20 tetrads).

The DNA sequence of the MSH5 gene was obtained by sequencing the majority of the $4.5-\mathrm{kb}$ BglII-HindIII complementing fragment as described in Materials and methods (Fig. 3). When the DNA sequence was used to search the GenBank data base, an exact match was found between one end of the fragment containing MSH5 and the last $161 \mathrm{bp}$ of the submitted sequence that contains the $C L B 3$ (cyclin B) gene (Fitch et al. 1992). The sequence of MSH5 is not present in the GenBank data base (see below). This fact, coupled with the mapping data, demonstrates that $\mathrm{MSH} 5$ represents a new gene.

To confirm that the cloned DNA represents $M S H 5$ and not a suppressor, a complementation test was performed between msh5::URA3 and msh5-1. Dissection of tetrads from a msh5::URA3/msh5-1 heteroallelic diploid gave $42.3 \%$ spore viability (55 tetrads), demonstrating that the cloned gene is MSH5.

\section{MSH5 encodes a MutS homolog}

The MSH5 open reading frame is composed of $2703 \mathrm{nu}$ cleotides and encodes a protein of 901 amino acids (predicted molecular mass $=102 \mathrm{kD}$ ). An $8 / 9$ match with the URS1 consensus sequence is located at position -99 relative to the MSH5-initiating methionine. This cis-acting element has been found upstream or within a number of meiotically regulated genes (for review, see Mitchell 1994); however, meiosis-specific expression of $\mathrm{MSH} 5$ has not yet been demonstrated.

When the Msh5p amino acid sequence was compared with other proteins in the GenBank data base (Altschul et al. 1990), strong homology to the MutS family of proteins was found (Fig. 4). Msh5p exhibits $23.5 \%$ identity and $49.0 \%$ similarity with the entire Escherichia coli MutS protein. Of the four previously identified yeast MutS homologs, Msh5p is most homologous to Msh2p (25.6\% identity, $50.3 \%$ similarity) and least homologous to $\mathrm{Msh} 4 \mathrm{p}$ (21.0\% identity, $45.6 \%$ similarity). As with the other members of the MutS family, the strongest regions of homology for Msh5p are located in the carboxy-terminal part of the protein. Two functional domains-a nucleotide-binding site and a helix-turn-helix DNA-binding domain-are both present in Msh5p. A consensus sequence for the nucleotide-binding site region is depicted in Figure 4. All four of the domains involved in NTP binding found in the superfamily of recombination/ repair proteins defined by Gorbalenya and Koonin (1990) are present in Msh5p. Also depicted is a comparison of the Msh5p helix-turn-helix region with the consensus sequence derived by Ross-MacDonald and Roeder (1994). The placement of the conserved domains within Msh5p is similar to that seen for the other members of the family.

Table 4. Effect of msh5::URA3 on gene conversion

\begin{tabular}{|c|c|c|c|c|}
\hline \multirow[b]{2}{*}{ Strain } & \multirow[b]{2}{*}{ Genotype } & \multicolumn{3}{|c|}{ Gene conversion $(\%)^{a}$} \\
\hline & & HIS4/his4-X & ARG4/arg4-hsp1 & leu2-K/leu2 $\Delta$ hisG \\
\hline & MSH5 & & & \\
\hline NH144 & $\begin{array}{l}\overline{M S H 5} \\
\text { msh5::URA3 }\end{array}$ & $5.1(297)$ & $1.7(293)$ & $2.6(193)$ \\
\hline NH145 & $\overline{m s h 5:: U R A 3}$ & $7.6(264)$ & $\underline{10.4}(259)$ & $3.5(114)$ \\
\hline
\end{tabular}

${ }^{a}$ Only four-viable spore tetrads were analyzed. For HIS4 and ARG4, gene conversions were scored as tetrads exhibiting $3^{+}: 1^{-}$or $3^{-}: 1^{+}$ segregation for the marker. For LEU2, the presence of a Leu ${ }^{+}$spore indicated gene conversion. A single PMS event $\left(5^{+}: 3^{-} \mid\right.$was observed for the arg4-nsp1 marker in NH144. The underlined value differs significantly from wild type $\left(\chi^{2}, P<0.001\right)$. Numbers in parentheses indicate the number of tetrads assayed. 
Hollingsworth et al.

Figure 3. DNA sequence of MSH5. The predicted Msh5 protein sequence is presented using the single-letter amino acid code below the corresponding nucleotide sequence. Nucleotides indicated by uppercase letters and underlined represent restriction sites (AGACTC, BglII; CTCGAG, Xhol). The lowercase bold triplet at position 7 represents the last codon of the $C L B 3$ gene. The bold uppercase letters at position 192 indicate the putative URS 1 element. The underlined amino acids represent the homologous MutS domains described in Fig. 4.

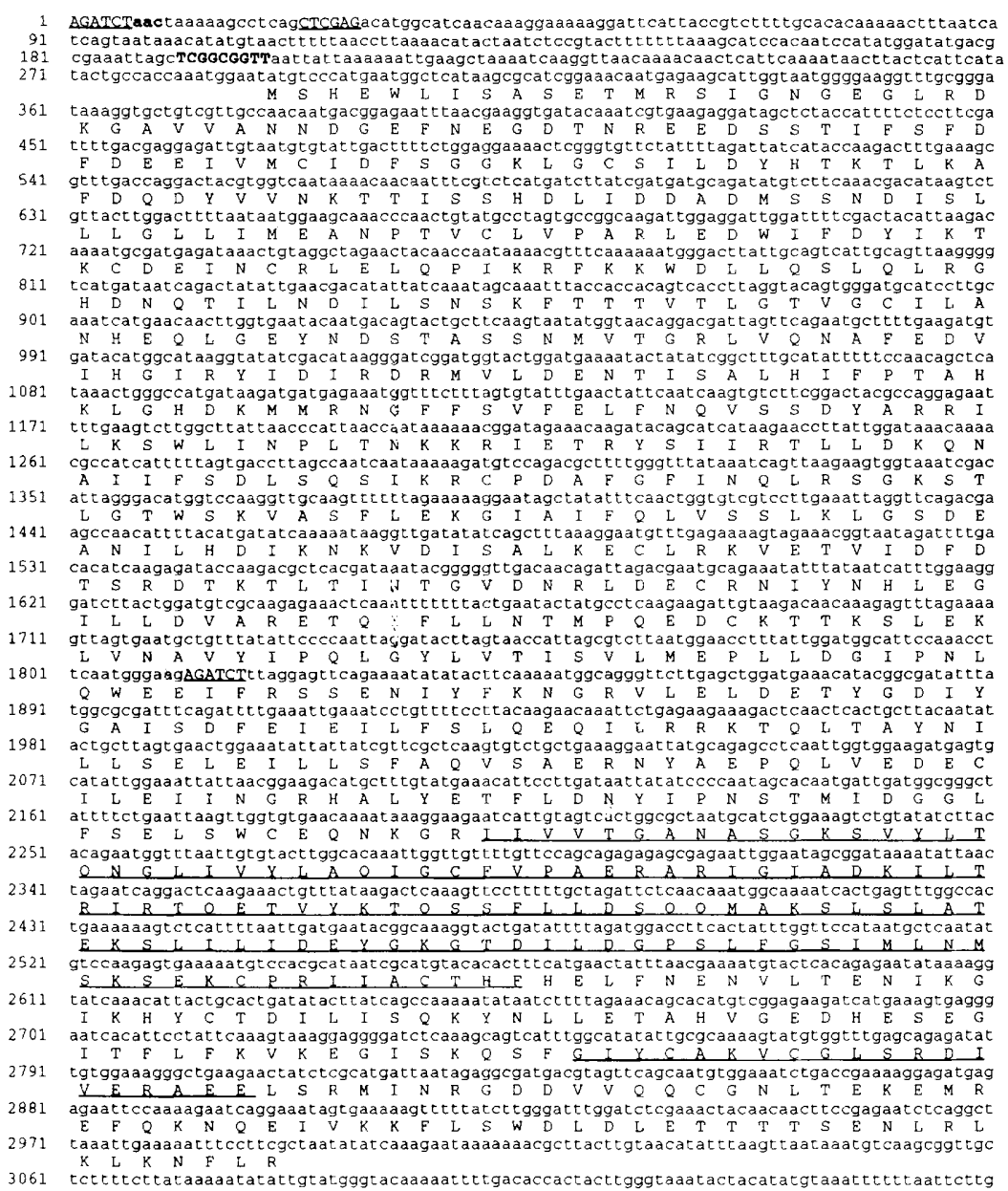

the spore undergoes the first mitotic division following meiosis. PMS is detected by the presence of half-sectored spore colonies. We disrupted MSH5 in the SK1 background used by Reenan and Kolodner (1992b) so that direct comparisons between $m s h 2$ and msh5::URA3 could be made. The resulting diploid (NH145) is heterozygous for the his4-X and arg4-nsp alleles. Although gene conversion freqencies at these loci are either the same or increased relative to the $M S H 5$ diploid NH144 (Table 4), no PMS events were observed at either ARG4 or HIS4 (see Materials and methods). In contrast, Reenan and Kolodner (1992b) reported that $46 \%$ of the gene conversion events involving the his4- $\mathrm{x}$ allele exhibited PMS in $m s h 2$ strains. The lack of PMS events for arg4-nsp was somewhat surprising given that this allele has been observed to exhibit moderate amounts of PMS in wild-type strains (Lichten et al. 1990, Ross-MacDonald and Roeder 1994). In our hands, the gene conversion frequency in NH144 (MSH5) is also signficantly reduced from that observed by other groups [e.g., $1.7 \%$ vs. $9.2 \%$ found by Ross-MacDonald and Roeder (1994)]. It is clear from these data, however, that the lack of PMS events in the msh5::URA3 diploid indicates that MSH5 is not required for mismatch repair during meiosis. 


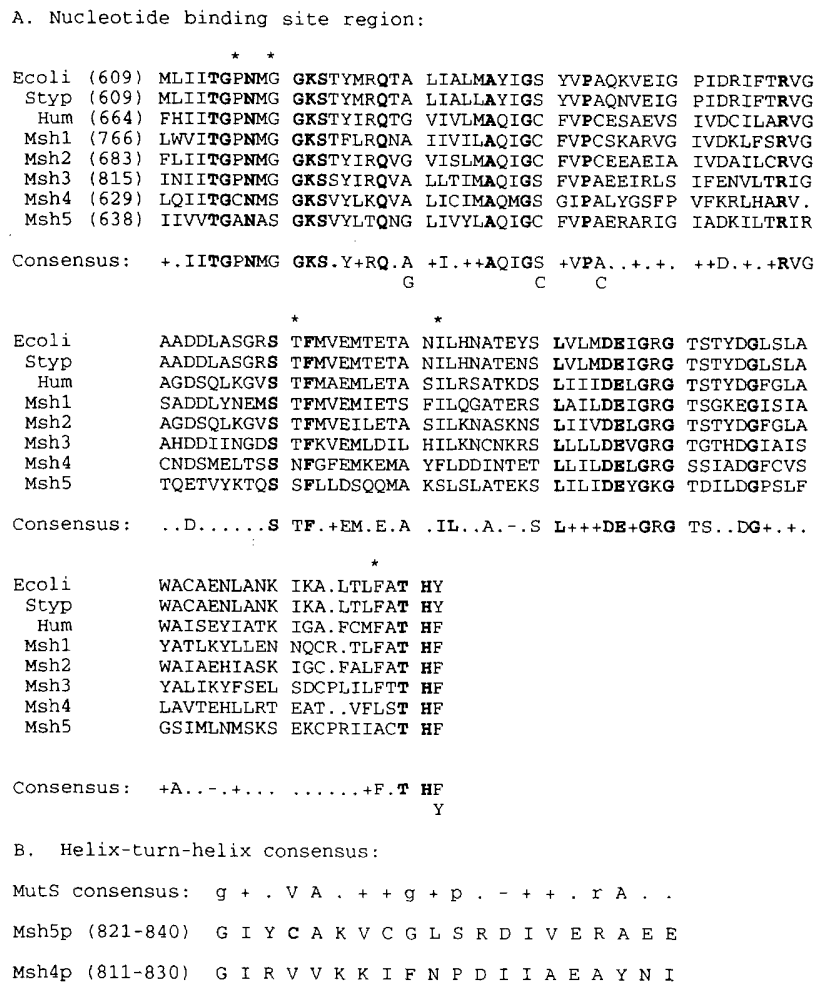

Figure 4. Alignment of Msh5 protein with MutS homologs known to be involved in mismatch repair and/or recombination. (A) Alignment of the region containing the 4 nucleotidebinding site motifs of Msh5 protein with seven other members of the MutS family. The consensus sequence was generated using the following parameters: A bold uppercase letter means that the amino acid is conserved in all family members; an uppercase letter means that the amino acid is found in at least six of eight members; $(+1$ a hydrophobic position; $(-1)$ a hydrophilic position; (.) no similarity. Positions bearing two letters mean that either amino acid may be present. Asterisks $\left({ }^{*}\right)$ mark amino acids that are conserved in all family members except Msh4 and Msh5 proteins (also designated Msh4p and Msh5p). Numbers in parentheses indicate the positions of the amino acids in the proteins. (Ecoli) E. coli MutS, Schlensog and Bock (1991); (Styp) S. typhmurium MutS, Haber et al. (1988); (Hum) hMSH2, Leach et al. (1993); (Msh1 and Msh2) Reenan and Kolodner (1992a); (Msh3) New et al. (1993); (Msh4) Ross-Macdonald and Roeder (1994). (B) Comparison of the Msh5 protein helix-turn-helix domain to the consensus defined by RossMacDonald and Roeder (1994). Amino acids found in all of the MutS members are uppercase, whereas those present in the majority of proteins are lowercase. $(+\mid$ A hydrophobic residue; $(-)$ a hydrophilic residue.

\section{MSH4 and MSH5 belong to the same genetic epistasis group}

MSH4 is a gene from S. cerevisiae that encodes a MutS homolog that also specifically affects meiotic reciprocal recombination and does not appear to have a role in mismatch repair (Ross-Macdonald and Roeder 1994). Mutants in either $\mathrm{MSH} 4$ or $\mathrm{MSH} 5$ decrease spore viability to $40 \%-60 \%$. The residual spore viability observed in $\mathrm{msh} 4$ and msh5 strains may result because (1) the two genes are functionally redundant and can partially substitute for each other, (2) the two genes act on separate pathways, either of which is capable of generating aproximately $50 \%$ viable spores, or $(3)$ the two genes function in the same process which, when abolished, decreases spore viability by half. If either of the first two cases is true, disruption of both genes in the same diploid should lead to a reduction in spore viability below the viability observed for either single mutant alone. Isogenic diploids were constructed that differ only at $\mathrm{MSH} 4$ and/or MSH5. These diploids were sporulated, and the resulting tetrads dissected to assess their spore viability. For the MSH4 MSH5 diploid NH144, spore viability was $97 \%$ (377 tetrads). The msh4::LEU2 msh5::URA3 diploid NH155 produced $41 \%$ viable spores (297 tetrads), similar to the $38 \%$ observed for msh5::URA3 alone (1370 tetrads). [Disruption of $\mathrm{MSH} 4$ in this background gives rise to $56 \%$ viable spores (Ross-Macdonald and Roeder 1994)]. Similar results were obtained when these genes were disrupted in the A364A strain background (N.M. Hollingsworth, unpubl.). NH155 was also analyzed for recombination between HIS4 and MAT. A threefold reduction in recombination similar to that found for msh5::URA3 was observed for the double mutant (Table 2). [msh4 reduced recombination twofold in this interval (Ross-MacDonald and Roeder 1994)]. MSH4 and MSH5 therefore belong to the same epistasis group both in terms of spore viability and recombination. Furthermore, it appears that $\mathrm{MSH} 5$ is epistatic to $\mathrm{MSH} 4$. One interpretation of these results is that $\mathrm{MSH} 4$ and $\mathrm{MSH} 5$ work in the same pathway to facilitate meiotic recombination between homologs.

\section{Discussion}

Using a screen specific for mutants defective in reciprocal crossing-over between homologs during meiosis in yeast, we have isolated new alleles of $H O P 1, R E D 1$, and $M E K 1$. The screen also identified MSH5, a gene encoding a novel member of the MutS family of proteins. MutS has been studied extensively in $E$. coli and has been shown to be a protein important for mismatch repair and for preventing recombination between partially diverged DNA sequences (homeologous recombination). MutS protein binds to DNA mismatches and then recruits a complex of other proteins including MutL and MutH to effect the repair of the mismatches (for review, see Modrich 1991). Recently a MutS homolog in humans has been implicated in the development of hereditary nonpolyposis colon cancer (Fishel et al. 1993; Leach et al. 1993).

Four other MutS homologs are known in S. cerevisiae. $M S H 1, M S H 2$, and $M S H 3$ were isolated by the polymerase chain reaction using primers directed against conserved regions of MutS (Reenan and Kolodner 1992a; New et al. 1993). MSH4 was identified by a transposon insertion that resulted in meiosis-specific lac $Z$ expression (Burns et al. 1994). MSH1 is targeted to mitochondria and is important for the stability of the mitochon- 
drial genome. MSH2 is involved in mismatch repair both in mitosis and meiosis and in the prevention of homeologous recombination (Reenan and Kolodner 1992b; Selva et al. 1995). MSH3 may have a small role in mismatch repair and in certain situations acts to inhibit homeologous recombination (Selva et al. 1995). MSH4 differs from the previously identified MutS homologs in that it plays no role in mismatch repair but is instead exclusively required for wild-type levels of reciprocal recombination during meiosis in yeast (Ross-Macdonald and Roeder 1994).

To test whether MSH5 is involved in mismatch repair we assayed two phenotypes characteristic of mismatch repair defective mutations-an increase in mutation rate in vegetatively growing cells and increased levels of postmeiotic segregation. In both cases, the msh 5 mutant was indistinguishable from the isogenic $\mathrm{MSH} 5$ control. Spore viability in the msh5 spo13 disomic haploid is slightly improved relative to wild type (N.M. Hollingsworth, unpubl.), indicating that lethal lesions are not being introduced during premeiotic DNA synthesis (Ross-Macdonald and Roeder 1994). Therefore, despite its strong homology to MutS, the role of MSHS is not in mismatch repair.

The MSH5 gene appears to be restricted to meiosis. No mitotic phenotypes have been observed so far in $\mathrm{msh} 5$ strains (MMS sensitivity, spontaneous reciprocal recombination, gene conversion, and growth rate have been examined; data not shown). Strains lacking MSH5 show an average threefold reduction in meiotic crossing-over between homologs measured on two different chromosomes. This defect in interhomolog reciprocal exchange is probably responsible for increased levels of Meiosis I nondisjunction and a reduction in spore viability. The role of $\mathrm{MSH} 5$ therefore appears to be in facilitating crossovers between homologs during meiosis.

The phenotypes observed for $m s h 5$ are similar to those found in strains lacking the meiosis-specific MutS homolog, MSH4. Interestingly, for spore viability and recombination, the MSH5 defect consistently seems to be slightly more severe. The msh5::URA3 allele was introduced into the same spo13 strains used to analyze recombination for $\mathrm{msh} 4$ and so direct comparisons may be made. In every interval the msh 5 mutant exhibited at least $10 \%$ less recombination than $m s h 4$ (Table 3; RossMacdonald and Roeder 1994). This result is consistent with the observation that the msh5::URA3 mutation results in fewer viable spores $(38 \%$ vs. $56 \%)$ than the $m s h 4$ disruption in the SK1 background.

Ross-Macdonald and Roeder (1994) have proposed that the twofold reduction in reciprocal recombination caused by $m s h 4$ may be attributable to the existence of two independent pathways of recombination, only one of which requires $\mathrm{MSH} 4$. We tested the idea that MSH5 might be involved in the $M S H 4$-independent pathway by assaying spore viability in a $\mathrm{msh} 4 \mathrm{msh} 5$ diploid. In two different strain backgrounds the number of viable spores in the double mutant was no lower than the more severe single mutant msh5. Recombination between HIS4 and $M A T$ in the double mutant was also not reduced beyond the level of $m s h 5$ alone. This result indicates that rather than working in separate pathways, MSH4 and MSH5 function in the same process. One model to account for this result would be if Msh4p and Msh5p interact to form heterodimers. If this is the case, the fact that $M S H 5$ is epistatic to MSH4 would require that MSH5 homodimers also be important. Ross-MacDonald and Roeder (1994) have noted that the DNA-binding domain of Msh4p differs in several conserved residues from the MutS family. Interestingly, these sites are much more conserved in Msh5p (Fig. 4). In the heterodimer model it may be the Msh5p subunit that is responsible for binding DNA while the Msh4 protein is important for interacting with other proteins. In support of the heterodimer model, Drummond et al. (1995) have shown recently that the human mismatch repair activity hMutS $\alpha$ is a heterodimer of MutS homologs.

The role of MutS in mismatch repair is to bind DNA duplexes containing mispaired bases ( $\mathrm{Su}$ and Modrich 1986). MutS protein therefore recognizes a structure as opposed to a specific sequence. The fact that a human MutS homolog, hMSH2, and yeast Msh2p are capable of binding mismatches comprised of loops of DNA as large as 14 nucleotides but $E$. coli MutS cannot (Fishel et al. 1994; Alani et al. 1995), suggests that during evolution different MutS family members have diverged to recognize different structures. Because $\mathrm{MSH} 4$ and $\mathrm{MSH} 5$ are not required for mismatch repair and because both exhibit a mutant phenotype in homothallic diploids where no mismatches exist during meiosis /Ross-Macdonald and Roeder 1994; N.M. Hollingsworth and C. Halsey, unpubl.), it seems likely that these proteins bind a DNA structure other than a mismatch.

MSH5 was isolated using a screen that demanded efficient intrachromosomal reciprocal recombination. Furthermore, we have demonstrated that gene conversion at LEU2 is not decreased in the same strains in which reciprocal crossing-over between HIS4 and MAT, two markers that flank LEU2, is reduced threefold. These results suggest that normal levels of heteroduplex are forming to allow for gene conversion but that the resolution of such intermediates is somehow biased against crossovers. One possibility is that Msh5p binds a configuration of DNA that is formed specifically between homologs. Perhaps binding of such substrates by Msh5p stabilizes recombination intermediates formed between non-sister chromatids (Collins and Newlon 1994, Schwacha and Kleckner 1994), thereby channeling the recombination mechanism to favor events between homologs.

\section{Materials and methods}

Yeast strains and media

The genotypes of strains used in this work are listed in Table 1. Standard yeast genetic methods were employed (Mortimer and Hawthorne 1969). Liquid and solid media have been described (Hollingsworth and Johnson 1993). All gene disruptions created by transformation were confirmed by Southern blot analysis. 


\section{Plasmid construction}

Plasmids for this study were constructed using standard procedures (Maniatis et al. 1982). pNH181 was constructed by delet ing first a HindIII fragment and then an SphI fragment from the original clone pMSH5-2. pNH180-13 was made by cloning a 4.4-kb HindIII fragment from pMSH5-2 into the HindIII site of YIp5. pNH180-13 was targeted to the MSH5 region of chromosome IV by digestion with XbaI (Orr-Weaver et al. 1981). pNH189-2 was generated by ligating a 4.3-kb HindIII-Xhol fragment that contains the entire MSH5 gene from pMSH5-2 into pVZl cut with HindIII and SalI. The msh5::URA3 insertion allele was constructed by cloning a $1.1-\mathrm{kb}$ BglII-BamHI fragment containing URA3 into the BglII site of pNH189-2. Digestion of the resulting plasmid, pNH190, with EcoRI and ClaI releases a $3.2-\mathrm{kb}$ fragment that can be used to replace the wildtype copy of the MSH5 by one-step gene transplacement (Rothstein 1983). pNH187-2 contains a 1.9-kb BglII fragment from $\mathrm{pNH} 18 \mathrm{l}$ cloned into the BamHI site of $\mathrm{pVZ1}$. The msh4::LEU2 disruption was generated using $\mathrm{p} 6 \mathrm{H}$ from $\mathrm{P}$. RossMacDonald (Yale University, New Haven, CT).

\section{The meiotic interhomolog recombination mutant screen}

The interhomolog recombination screen has been described previously in detail in Hollingsworth and Byers (1989). The screen was performed with some minor modifications. The disomic haploid karC2-4 was mutagenized with ethylmethane sulfonate in two independent rounds at $23^{\circ} \mathrm{C}$. Time points exhibiting $15 \%$ cell viability were used.

Of 9855 patched colonies, 243 were initially scored as having elevated levels of $\mathrm{His}^{+} \mathrm{Leu}^{+} \mathrm{Cyh}^{\mathrm{T}}$ cells. Failure to retest was observed in 114 candidates, whereas 59 were either increased for intrachromosomal exchange in vegetative cells or had a mutant cyh2 allele now resident in the duplication and were therefore discarded.

The remaining 70 candidates were analyzed further in two ways. First the frequency of intrachromosomal recombination in both mitotic and meiotic cells was quantitated as described in Hollingsworth and Byers (1989) to ensure that the effect was meiosis-specific. Second, dyads from the sporulated disomic haploid strains were dissected to determine the percent spore viability. The segregation of markers on chromosome III was scored in the dyads to assess the effects of the mutations on equational versus reductional segregation and on recombination between homologs.

To determine the spore viability of the mutants in a SPO13 meiosis, SPO13 diploids homozygous for each mutation were constructed. Haploid derivatives of both mating types were obtained as described in Hollingsworth and Byers (1989). The MATa derivatives were converted to $\mathrm{Spol}^{+}$by the integration of the SPO13 gene (Hollingsworth and Byers 1989). Afer crossing the two haploids, the resulting diploids were sporulated and dissected to ascertain the number of viable spores.

\section{Cloning the MSH5 gene}

The MSH5 gene was cloned by complementation of the msh5-1 spore inviability phenotype using the random spore method described in Hollingsworth and Byers (1989). The msh5-1 NH111 diploid was transformed with a genomic yeast DNA library constructed in YCp50 (Rose et al. 1987). Two different plasmids with overlapping inserts of 17 and $11.6 \mathrm{~kb}$ /designated pMSH5-1 and pMSH5-2) were found to complement msh5-1. The smallest MSH5 complementing activity was found to reside in a $4.7-\mathrm{kb}$ HindIII-SphI fragment.

\section{Mapping MSH5}

The MSH5 gene was placed on the physical map of the $S$. cerevisiae genome by hybridizing fragments from either pMSH5-1 or pMSH5-2 (0.9- and 1.9-kb SphI fragments, respectively) to a set of overlapping genomic fragments contained in a series of $\lambda$ clones (available from the American Type Culture Collection). Both fragments identified the same two $\lambda$ clones (ATCC no. 71144 and 70585 ), indicating that the two inserts carry overlapping sequences. These $\lambda$ clones are adjacent to one carrying $C D C 36$, leading to the prediction that tight genetic linkage should be observed between MSH5 and CDC36. The MSH5 region was marked with $U R A 3$ using pNH180-13 and crossed to a ura3 cdc36 strain. Tetrad analysis of the resulting diploid demonstrated that $\mathrm{MSH} 5$ and $C D C 36$ are $\sim 1.9 \mathrm{cM}$ apart [50PD: ONPD: 2T, map distance calculated using the formula of Perkins (1949)].

\section{Sequencing MSH5}

The DNA sequence of the MSH5 gene was obtained by first constructing exonuclease III deletion derivatives of plasmids pNH187-2 and pNH189-2 (Henikoff 1984). These plasmids were used as templates for the dideoxy method of DNA sequencing using T7 or T3 primers (Sanger et al. 1977). To fill in gaps in the sequence not covered by the deletion series, specific oligonucleotides were synthesized and used as primers. The DNA sequence of both strands was determined. The GenBank accession number is $\mathbf{L} 42517$.

\section{Mutation rate analysis}

Ten independent single colonies of PS593 or PS593/190 were inoculated into YEPD and grown at $30^{\circ} \mathrm{C}$ to saturation. The cells were plated onto YPAD to obtain the viable cell titer and onto - Arg + Can plates to determine the number of spontaneously arising $\operatorname{Can}^{\mathrm{r}}$ mutants. The mutation rates were calculated as described in Ivanov et al. (1992).

\section{Gene conversion and PMS analysis}

Tetrads were dissected onto YPAD plates, and the spore colonies transferred by replica plating to appropriate dropout media. To ensure that we could detect PMS events, prototrophic and auxotrophic spores were micromanipulated adjacent to each other and the resulting colonies were tested as described. In such cases, sectoring was readily observed.

\section{Acknowledgments}

We thank Breck Byers, Neta Dean, Joanne Engebrecht, and Aaron Neiman for helpful comments on the manuscript. Sylvia Sanders provided the $c d c 36$ strain. Petra Ross-Macdonald generously provided both unpublished information and reagents. N.M.H. thanks Sandy Johnson and members of his laboratory for their interest and support during the early part of this work. This work was supported in part by National Institutes of Health (NIH) grant GM44532 to A.D.J. and by NIH grant GM50717 to N.M.H. N.M.H. was supported in part by the American Cancer Society /California Division Fellowship S-29. 91).

The publication costs of this article were defrayed in part by payment of page charges. This article must therefore be hereby marked "advertisement" in accordance with 18 USC section 1734 solely to indicate this fact. 


\section{References}

Alani, E., N.-W. Chi, and R. Kolodner. 1995. The Saccharomyces cerevisiae Msh2 protein specifically binds to duplex oligonucleotides containing mismatched DNA base pairs and insertions. Genes \& Dev. 9: 234-247.

Altschul, S.F., W. Gish, W. Miller, E.W. Myers, and D.J. Lipman. 1990. Basic local alignment search tool. I. Mol. Biol. 215: $403-410$.

Burns, N., B. Grimwade, P.B. Ross-Macdonald, E.-Y. Choi, K. Finberg, G.S. Roeder, and M. Snyder. 1994. Large-scale analysis of gene expression, protein localization, and gene disruption in Saccharomyces cerevisiae. Genes \& Dev. 8: 1087-1105.

Collins, I. and C.S. Newlon. 1994. Meiosis-specific formation of joint DNA molecules containing sequences from homologous chromosomes. Cell 76: 65-75.

Drummond, J.T., G-M. Li, M.J. Longley, and P. Modrich. 1995. Isolation of an hMSH2-pl60 heterodimer that restores DNA mismatch repair to tumor cells. Science (in press).

Engebrecht, J. and G.S. Roeder. 1989. Yeast mer1 mutants display reduced levels of meiotic recombination. Genetics 121: $237-247$.

Engebrecht, J., J. Hirsch, and G.S. Roeder. 1990. Meiotic gene conversion and crossing over: their relationship to each other and to chromosome synapsis and segregation. Cell 62: 927-937.

Fishel, R., M.K. Lescoe, M.R.S. Rao, N.G. Copeland, N.A. Jenkins, J. Garber, M. Kane, and R. Kolodner. 1993. The human mutator gene homolog MSH2 and its association with hereditary nonpolyposis colon cancer. Cell 75: 1027-1038.

Fishel, R., A. Ewel, L. Suman, M.K. Lescoe, and J. Griffith. 1994. Binding of mismatched microsatellite DNA sequences by the Human MSH2 protein. Science 266: 1403-1 405.

Fitch, I., C. Dahmann, U. Surana, A. Amon, K. Nasmyth, L. Goetsch, B. Byers, and B. Futcher. 1992. Characterization of four B-type cyclin genes of the budding yeast Saccharomyces cerevisiae. Mol. Cell. Biol. 3: 805-818.

Gorbalenya, A.E. and E.V. Koonin. 1990. Superfamily of UvrArelated NTP-binding proteins: Implications for rational classification of recombination/repair systems. /. Mol. Biol. 213: 583-591.

Gottlieb, S., J. Wagstaff, and R.E. Esposito. 1989. Evidence for two pathways of meiotic intrachromosomal recombination in yeast. Proc. Natl. Acad. Sci. 86: 7072-7076.

Haber, L.T., P.P. Pang, D.I. Sobell, J.A. Mankovich, and G.C. Walker. 1988. Nucleotide sequence of the Salmonella typhimurium mutS gene required for mismatch repair: Homology of MutS and HexA of Streptococcus pneumoniae. I. Bacteriol. 170: 197-202.

Hawley, R.S. 1987. Exchange and chromosomal segregation in eukaryotes. In Meiosis (ed. P.B. Moens), pp. 497-527. Academic Press, New York.

Henikoff, S. 1984. Unidirectional digestion with exonuclease III creates targeted breakpoints for DNA sequencing. Gene 28: 351-359.

Hollingsworth, N.M. and B. Byers. 1989. HOP1: A yeast meiotic pairing gene. Genetics 121: 445-462.

Hollingsworth, N.M. and A.D. Johnson. 1993. A conditional allele of the Saccharomyces cerevisiae HOP1 gene is suppressed by overexpression of two other meiosis-specific genes: RED1 and REC104. Genetics 133: 785-797.

Hollingsworth, N.M., L. Goetsch, and B. Byers. 1990. The HOP1 gene encodes a meiosis-specific component of yeast chromosomes. Cell 61: 73-84.

Ivanov, E.L., V.G. Korolev, and F. Fabre. 1992. XRS2, a DNA repair gene of Saccharomyces cerevisiae, is needed for meiotic recombination. Genetics 132: 651-664.

Leach, F.S., N.C. Nicolaides, N. Papadopoulos, B. Lui, J. Jen, R. Parsons, P. Peltomaki, P. Sistonen, L.A. Aaltonen, M. Nystrom-Lahti, et al. 1993. Mutations of a mutS homolog in hereditary monpolyposis colorectal cancer. Cell 75: 12151225.

Leem, S.-H. and H. Ogawa. 1992. The MRE4 gene encodes a novel protein kinase homologue required for meiotic recombination in Saccharomyces cerevisiae. Nucleic Acids Res. 20: $449-457$.

Lichten, M., C. Goyon, N.P. Schultes, D. Treco, J.W. Szostak, J.E. Haber, and A. Nicolas. 1990. Detection of heteroduplex DNA molecules among the products of Saccharomyces cerevisiae meiosis. Proc. Natl. Acad. Sci. 87: 7653-7657.

Maniatis, T., E.F. Fritsch, and J. Sambrook. 1982. Molecular cloning: A laboratory manual. Cold Spring Harbor Laboratory, Cold Spring Harbor, New York.

Meuwissen, R.L.J., H.H. Offenberg, A.J.J. Dietrich, A. Riesewijk, M. van Iersel, and C. Heyting. 1992. A coiled-coil related protein specific for synapsed regions of meiotic prophase chromosomes. EMBO I. 11: 5091-5100.

Mitchell, A.P. 1994. Control of meiotic gene expression in Sac charomyces cerevisiae. Microbiol. Rev. 58: 56-70.

Miyazaki, W.Y. and T.L. Orr-Weaver. 1992. Sister-chromatid misbehavior in Drosophila ord mutants. Genetics 342: 1047-1061.

Modrich, P. 1991. Mechanisms and biological effects of mismatch repair. Annu. Rev. Genet. 229-253.

Mortimer, R.K. and D.C. Hawthorne. 1969. Yeast genetics. In The yeasts (ed. A.H. Rose and J.S. Harrison), pp. 385-360. Academic Press, New York.

New, L., K. Liu, and G.F. Crouse. 1993. The yeast gene MSH3 defines a new class of eukaryotic MutS homologues. Mol. Gen. Genet. 239: 97-108.

Orr-Weaver, T.L., J.W. Szostak, and R.J. Rothstein. 1981. Yeast transformation: A model system for the study of recombination. Proc. Natl. Acad. Sci. 78: 6354-6358.

Perkins, D.D. 1949. Biochemical mutants in the smut fungus Ustilago maydis. Genetics 34: 607-626.

Petes, T.D., R.E. Malone, and L.S. Symington. 1991. Recombination in yeast. In The molecular and cellular biology of the yeast Saccharomyces: Genome dynamics, protein synthesis and energetics (ed. J.R. Broach, J.R. Pringle, and E.W. Jones), pp. 407-522.

Rayssiguier, C., D.S. Thaler, and M. Radman. 1989. The barrier to recombination between Escherichia coli and Salmonella typhimurium is disrupted in mismatch-repair mutants. $\mathrm{Na}$ ture 342: 396-401.

Reenan, R.A.G. and R.D. Kolodner. 1992a. Isolation and characterization of two Saccharomyces cerevisiae genes encoding homologs of the bacterial HexA and MutS mismatch repair proteins. Genetics 132: 963-973.

- 1992b. Characterization of insertion mutations in the Saccharomyces cerevisiae MSH1 and MSH2 genes: Evidence for separate mitochondrial and nuclear functions. Genetics 132: 975-985.

Rockmill, B. and G.S. Roeder. 1990. Meiosis in asynaptic yeast. Genetics 126: 563-574.

- 1991. A meiosis-specific protein kinase homolog required for chromosome synapsis and recombination. Genes \& Dev. 5: 2392-2404.

- 1994. The yeast med1 mutant undergoes both meiotic homolog nondisjunction and precocious separation of sister chromatids. Genetics 136: 65-74.

Rose, M.D., P. Novick, J.H. Thomas, D. Botstein, and G.R. Fink. 
1987. A Saccharomyces cerevisiae genomic plasmid bank based on a centromere-containing shuttle vector. Gene 60: 113-124.

Ross-Macdonald, P. and G.S. Roeder. 1994. Mutation of a meiosis-specific MutS homolog decreases crossing over but not mismatch correction. Cell 79: 1069-1080.

Rothstein, R. 1983. One-step gene disruption in yeast. Methods Enzymol. 101: 202-209.

Sanger, F., S. Nicklen, and A.R. Coulson. 1977. DNA sequencing with chain terminating inhibitors. Proc. Natl. Acad. Sci. 74: 5463-5467.

Schlensog, V. and A. Bock. 1991. The Escherichia coli $f d v$ gene probably encodes MutS and is located at minute 58.8 adjacent to the hyc-hyp gene cluster. I. Bacteriol. 173: 7414 7415.

Schwacha, A. and N. Kleckner. 1994. Identification of joint molecules that form frequently between homologs but rarely between sister chromatids. Cell 76: 51-63.

Selva, E.M., L. New, G.F. Crouse, and R.S. Lahue. 1995. Mismatch correction acts as a barrier to homeologous recombination in Saccharomyces cerevisiae. Genetics 139: 11751188.

Su, S.-S. and P. Modrich. 1986. Escherichia coli mutS-encoded protein binds to mismatched DNA base pairs. Proc. Natl. Acad. Sci. 83: 5057-5061.

Sym, M. and G.S. Roeder. 1994. Crossover interference is abolished in the absence of a synaptonemal complex protein. Cell 19: 283-292.

Sym, M., J. Engebrecht, and G.S. Roeder. 1993. ZIP1 is a synaptonemal complex protein required for meiotic chromosome synapsis. Cell 72: 365-378.

Wagstaff, J.E., S. Klapholz, C.S. Waddell, L. Jensen, and R.E. Esposito. 1985. Meiotic exchange within and between chromosomes requires a common rec function in Saccharoymces cerevisiae. Mol. Cell. Biol. 5: 3532-3544. 


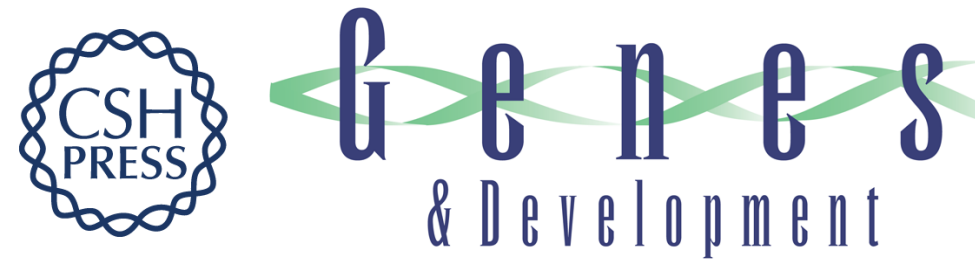

\section{MSH5, a novel MutS homolog, facilitates meiotic reciprocal recombination between homologs in Saccharomyces cerevisiae but not mismatch repair.}

N M Hollingsworth, L Ponte and C Halsey

Genes Dev. 1995, 9:

Access the most recent version at doi:10.1101/gad.9.14.1728

References This article cites 43 articles, 23 of which can be accessed free at:

http://genesdev.cshlp.org/content/9/14/1728.full.html\#ref-list-1

License

Email Alerting Service

Receive free email alerts when new articles cite this article - sign up in the box at the top right corner of the article or click here.

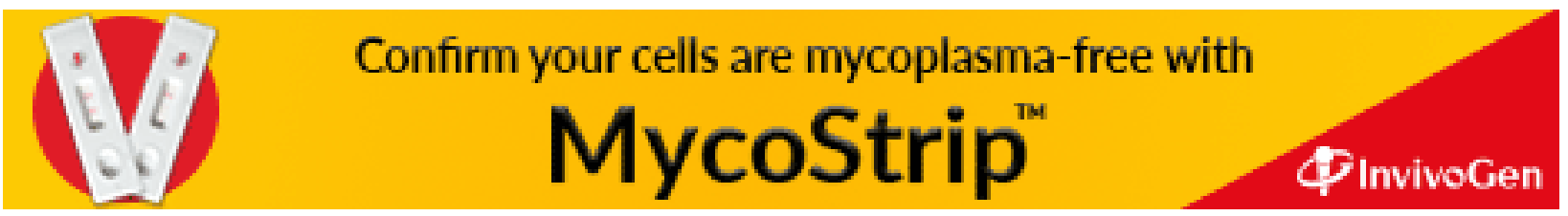

